\title{
A NOTE ON UNCONDITIONAL BASES
}

J. R. RETHERFORD ${ }^{1}$

1. Definitions and remarks. A sequence of nontrivial subspaces $\left\{M_{i}\right\}$ of a Banach space $X$ is a basis of subspaces for $X$ if and only if for every $x \in X, x$ can be uniquely written

$$
x=\sum_{1}^{\infty} x_{i}, \quad x_{i} \in M_{i} .
$$

If $\left\{M_{i}\right\}$ is a basis of subspaces for $X$, then the linear operators defined by $E_{i}(x)=x_{i}$, where $x=\sum_{1}^{\infty} x_{i}, x_{i} \in M_{i}$, form a sequence of orthogonal projections $\left(E_{i}=E_{i}^{2} ; E_{i} E_{j}=0, i \neq j\right)$. If $R\left(E_{i}\right)$ denotes the range of $E_{i}$, then clearly $R\left(E_{i}\right)=M_{i}$ and each $x \in X$ can be expressed

$$
x=\sum_{1}^{\infty} E_{i}(x) .
$$

If the convergence in (1.2) is unconditional, we say that $\left\{R\left(E_{i}\right)\right\}$ is an unconditional basis of subspaces for $X$. If $E_{i}$ is continuous for each $i$, we say the basis is a Schauder basis of subspaces.

Indeed, if $\left\{x_{i}\right\}$ is a basis (of vectors) for $X$ and $\left\{f_{i}\right\}$ the sequence of biorthogonal coefficient functionals, then the one-dimensional subspaces spanned by $x_{i}, i=1,2, \cdots$, form a basis of subspaces for $X$ and the induced projections, $E_{i}(x)=f_{i}(x) x_{i}$, are necessarily continuous.

We assume throughout this note that $\left\{E_{i}\right\}$ is a sequence of continuous orthogonal projections and that $X=\left[\bigcup_{i=1}^{\infty} R\left(E_{i}\right)\right]$, the linear closure of $\bigcup_{i=1}^{\infty} R\left(E_{i}\right)$. Thus all bases will be Schauder and in particular we will confine our attention to the unconditional Schauder bases of subspaces (u-bases) for $X$.

If $\Sigma$ denotes the collection of all finite subsets of the positive integers $\omega$, directed by inclusion, let

$$
\begin{aligned}
& U_{0}=\left\{x: \lim _{\sigma \in \Sigma} \sum_{i \in \sigma} E_{i}(x)=x\right\}, \quad U_{1}=\left\{x: \sup _{\sigma \in \Sigma}\left\|\sum_{i \in \sigma} E_{i}(x)\right\|<\infty\right\}, \\
& U_{2}=\left\{x: \lim _{\sigma \in \Sigma}\left\|\sum_{i \in \sigma} E_{i}(x)\right\|=\infty\right\} \text { and } U_{3}=X \backslash U_{1} .
\end{aligned}
$$

It is clear that $\left\{R\left(E_{i}\right)\right\}$ is a u-basis if and only if $X=U_{0}$. It is also clear that $U_{0} \subset U_{1}, U_{2} \subset U_{3}$ and $U_{0}$ is everywhere dense in $X$.

Received by the editors March 1, 1963 and, in revised form, August 2, 1963.

1 This research was supported by the Air Force Office of Scientific Research. 
The theories of ordered and unconditional bases are, generally speaking, parallel. The purpose of this paper is to show that $\left\{R\left(E_{i}\right)\right\}$ is a $u$-basis if and only if $U_{2}=U_{3}$ (the analogous result is known [6, Theorem 3, p. 1067] in the one-dimensional ordered case) and to show (contrary to the analogous statement for the ordered case [3, Theorem 3, p. 288]) that $U_{0}$ can indeed equal $U_{1}$ without $\left\{R\left(E_{i}\right)\right\}$ being a u-basis.

The author would like to thank the referee for his comments. In particular, the original proof of the theorem of $\$ 2$ has been considerably simplified by the referee.

2. The condition $U_{2}=U_{3}$. From $[4$, Theorem 6, p. 752] it follows that $\left\{R\left(E_{i}\right)\right\}$ is a $\mathrm{u}$-basis if and only if

$$
X=U_{1},
$$

and, if and only if

(2.2) there is a $k \geqq 1$ such that

$$
\left\|\sum_{i \in \sigma} x_{i}\right\| \leqq K\left\|\sum_{i \in \sigma^{\prime}} x_{i}\right\|
$$

whenever $\sigma^{\prime} \in \Sigma, \sigma \subseteq \sigma^{\prime}$ and $x_{i} \in R\left(E_{i}\right)$ for each $i \in \sigma$.

Theorem. $\left\{R\left(E_{i}\right)\right\}$ is a $u$-basis for $X$ if and only if $U_{2}=U_{3}$.

Proof. Clearly $X=U_{1}$ if and only if $U_{2}=U_{3}=\varnothing$. Thus by (2.1) if $\left\{R\left(E_{i}\right)\right\}$ is a u-basis, $U_{2}=U_{3}=\varnothing$. Conversely, suppose $X \neq U_{1}$ and thus $U_{3} \neq \varnothing$. Let $T(n)=\left\{x: \sup _{\sigma \in \Sigma}\left\|\sum_{i \in \sigma} E_{i}(x)\right\| \leqq n\right\}$. Then $U_{1}$ $=\bigcup_{n=1}^{\infty} T(n)$ and each $T(n)$ is closed. If some $T(n)$ were somewhere dense, then (2.2) would be satisfied and $U_{3}=\varnothing$. Thus $U_{3}$ is of the second category. Also, if $F(\sigma)=\left\{x:\left\|\sum_{i \in \sigma^{\prime}} E_{i}(x)\right\| \geqq\|x\|+1\right.$ if $\left.\sigma \subseteq \sigma^{\prime}\right\}$, then $F(\sigma)$ is closed. Since $U_{0}$ is everywhere dense, $F(\sigma)$ is nowhere dense, and since $U_{2} \subset U_{\sigma \in \Sigma} F(\sigma), U_{2}$ is of the first category.

3. The condition $U_{0}=U_{1}$. V. I. Gurarii [5, Theorem 5, p. 1029] has shown that in every infinite-dimensional Banach space there is an infinite-dimensional subspace with a conditional basis of vectors (i.e., a basis which is not unconditional). Let $X$ be a weakly complete Banach space and let $Y$ be a subspace of $X$ with a conditional basis $\left\{x_{n}\right\}$; let $\left\{E_{i}\right\}$ be the induced sequence of projections. $Y$ is weakly complete and it follows from $[2,4$ b, p. 60] and a well-known theorem of Orlicz (if $Y$ is weakly complete every weakly unconditionally convergent series is unconditionally convergent) that $U_{0}=U_{1}$. Clearly $\left\{R\left(E_{i}\right)\right\}$ is not a u-basis for $Y$. 


\section{BIBLIOGRAPHY}

1. S. Banach, Theorie des operations lineaires, Monogr. Mat., Tom I, Warsaw, 1932.

2. M. M. Day, Normed linear spaces, Springer-Verlag, Berlin, 1958.

3. M. M. Grinblyum, Sur la theorie des systèmes biorthogonaux, C. R. (Doklady) Acad. Sci. URSS (N. S.) 55 (1947), 287-290.

4. - On the representation of a space of type $B$ in the form of a direct sum of subspaces, Dokl. Akad. Nauk. SSSR 70 (1950), 749-752.

5. V. I. Gurarii, Inclinations of subspaces and conditional bases in Banach spaces, Soviet Math. Dokl. 3 (1962), 1028-1030.

6. S. R. Foguel, Biorthogonal systems in Banach spaces, Pacific J. Math. 7 (1957) 1065-1068.

Florida State University 